\title{
Optimal consumption of the stochastic Ramsey problem for non-Lipschitz diffusion
}

\section{Chuandi Liu*}

\section{"Correspondence:}

liuchuandi@swu.edu.cn

School of Mathematics and

Statistics, Southwest University,

Chongqing, 400715, China

\begin{abstract}
The stochastic Ramsey problem is considered in a growth model with the production function of a Cobb-Douglas form. The existence of a unique classical solution is proved for the Hamilton-Jacobi-Bellman equation associated with the optimization problem. A synthesis of the optimal consumption policy in terms of its solution is proposed.

MSC: 49L20; 49L25; 91B62
\end{abstract}

Keywords: Hamilton-Jacobi-Bellman equation; viscosity solutions; Ramsey problem; Cobb-Douglas production function

\section{Introduction}

We are concerned with the stochastic Ramsey problem in a growth model discussed by Merton [1]. For recent contribution in this direction, we refer to [2]. A firm produces goods according to the Cobb-Douglas production function $x^{\gamma}$ for capital $x$, where $0<\gamma<1$ (cf. Barro and Sala-i-Martin [3]). The stock of capital $X_{t}$ at time $t$ is modeled by the stochastic differential equation

$$
d X_{t}=X_{t}^{\gamma} d t+\sigma X_{t} d B_{t}, \quad t>0, X_{0}=x>0, \sigma \neq 0,
$$

on a complete probability space $(\Omega, \mathcal{F}, P)$ carrying a standard Brownian motion $\left\{B_{t}\right\}$ endowed with the natural filtration $\mathcal{F}_{t}$ generated by $\sigma\left(B_{s}, s \leq t\right)$.

The capital stock can be consumed and the flow of consumption at time $t$ is assumed to be written as $c_{t} X_{t}$. The rate of consumption $c=\left\{c_{t}\right\}$ per capital stock is called an admissible policy if $\left\{c_{t}\right\}$ is an $\left\{\mathcal{F}_{t}\right\}$-progressively measurable process such that

$$
0 \leq c_{t} \leq 1 \text { for all } t \geq 0 \text { a.s. }
$$

We denote by $\mathcal{A}$ the set of admissible policies. Given a policy $c \in \mathcal{A}$, the capital stock process $\left\{X_{t}\right\}$ obeys the equation

$$
d X_{t}=\left[X_{t}^{\gamma}-c_{t} X_{t}\right] d t+\sigma X_{t} d B_{t}, \quad X_{0}=x>0 .
$$

The objective is to find an optimal policy $c^{*}=\left\{c_{t}^{*}\right\}$ so as to maximize the expected discounted utility of consumption

$$
J_{x}(c)=E\left[\int_{0}^{\infty} e^{-\alpha t} U\left(c_{t} X_{t}\right) d t\right]
$$


over $c \in \mathcal{A}$, where $\alpha>0$ is a discount rate and $U(x)$ is a utility function in $C^{2}(0, \infty) \cap$ $C[0, \infty)$, which is assumed to have the following properties:

$$
U^{\prime}(\infty)=U(0)=0, \quad U^{\prime}(0+)=U(\infty)=\infty, \quad U^{\prime \prime}<0 .
$$

The Hamilton-Jacobi-Bellman (HJB for short) equation associated with this problem is given by

$$
\alpha u(x)=\frac{1}{2} \sigma^{2} x^{2} u^{\prime \prime}(x)+x^{\gamma} u^{\prime}(x)+\tilde{U}\left(x, u^{\prime}(x)\right), \quad x>0,
$$

where

$$
\tilde{U}(x, y)=\max _{0 \leq c \leq 1}\{U(c x)-c x y\} \quad \text { for } x, y>0 .
$$

This kind of economic growth problem has been studied by Kamien and Schwartz [4] and Sethi and Thompson [5, Chapter 11]. However, the optimization problem is unsolved. It is not guaranteed that (1.2) admits a unique positive solution $\left\{X_{t}\right\}$ and the value function is a viscosity solution of the HJB equation. The main difficulty stems from the fact that (1.5) is a degenerate nonlinear equation of elliptic type with the non-Lipschitz coefficient $x^{\gamma}$ in $(0, \infty)$. It is also analytically studied by [6], nevertheless in the finite time horizon. The resulting HJB equation is a parabolic partial differential equation (PDE, for short), which is very different from the elliptic PDE dealt with in the present paper.

In this paper, we provide the existence results on a unique positive solution $\left\{X_{t}\right\}$ to (1.2) and a classical solution $u$ of (1.5) by the theory of viscosity solutions. For the detail of the theory of viscosity solutions, we mention the works [7, 8] and [9]. An optimal policy is shown to exist in terms of $u$.

This paper is organized as follows. In Section 2, we show that (1.2) admits a unique positive solution. In Section 3, we show the existence of a viscosity solution $u$ of the HJB equation (1.5). Section 4 is devoted to the $C^{2}$-regularity of its solution. In Section 5, we present a synthesis of the optimal consumption policy.

\section{Preliminaries}

In this section, we show the existence of a unique solution $\left\{X_{t}\right\}$ to (1.2).

Proposition 2.1 There exists a unique positive solution $\left\{X_{t}\right\}=\left\{X_{t}^{x}\right\}$ to (1.2), for each $c \in \mathcal{A}$, such that

$$
\begin{aligned}
& E\left[X_{t}\right] \leq\left\{(1-\gamma) t+x^{1-\gamma}\right\}^{1 /(1-\gamma)}, \\
& E\left[X_{t}^{2}\right] \leq e^{\sigma^{2} t}\left\{2(1-\lambda) t+x^{2(1-\lambda)}\right\}^{1 /(1-\lambda)}, \quad \lambda:=(1+\gamma) / 2, \\
& \forall \varepsilon>0, \exists C_{\varepsilon}>0 \quad \text { s.t. } E\left[\left|X_{t}^{x}-X_{t}^{y}\right|\right] \leq C_{\varepsilon}|x-y|+\varepsilon\left(1+t^{1 /(1-\gamma)}+x+y\right), \quad x, y>0 .
\end{aligned}
$$

Proof We set $x_{t}=X_{t}^{1-\gamma}$. Then, by Ito's formula and (1.2),

$$
\begin{aligned}
d x_{t} & =(1-\gamma) X_{t}^{-\gamma} d X_{t}+\frac{\sigma^{2}}{2}(1-\gamma)(-\gamma) X_{t}^{1-\gamma} d t \\
& =(1-\gamma)\left[1-\left(c_{t}+\frac{\sigma^{2}}{2} \gamma\right) x_{t}\right] d t+(1-\gamma) \sigma x_{t} d B_{t}, \quad x_{0}=x^{1-\gamma} .
\end{aligned}
$$


By linearity, (2.4) has a unique solution $\left\{x_{t}\right\}$. Since

$$
d \hat{x}_{t}=(1-\gamma)\left[-\left(c_{t}+\frac{\sigma^{2}}{2} \gamma\right) \hat{x}_{t}\right] d t+(1-\gamma) \sigma \hat{x}_{t} d B_{t}, \quad \hat{x}_{0}=x^{1-\gamma}
$$

has a positive solution $\left\{\hat{x}_{t}\right\}$, we see by the comparison theorem [10, Chapter 6 , Theorem 1.1] that $x_{t} \geq \hat{x}_{t}>0$ holds almost surely (a.s.). Therefore, (1.2) admits a unique positive solution $\left\{X_{t}\right\}$ of the form $X_{t}=x_{t}^{1 /(1-\gamma)}$, which satisfies $\sup _{0 \leq t \leq T} E\left[X_{t}^{4}\right]<\infty$ for each $T \geq 0$.

Let $\theta_{t}$ be the right-hand side of (2.1) and $\phi_{t}=E\left[X_{t}\right]$. Obviously, we see that $\theta_{t}$ is a unique solution of

$$
d \theta_{t}=\theta_{t}^{\gamma} d t, \quad \theta_{0}=x>0
$$

By (1.2) and Jensen's inequality,

$$
d \phi_{t}=d E\left[X_{t}\right]=E\left[X_{t}^{\gamma}-c_{t} X_{t}\right] d t \leq \phi_{t}^{\gamma} d t
$$

Since $\theta_{0}=\phi_{0}=x$, we deduce $\phi_{t} \leq \theta_{t}$, which implies (2.1).

Similarly, let $\Theta_{t}$ be the right-hand side of (2.2) and $\Phi_{t}=E\left[X_{t}^{2}\right]$. By substitution, it is easy to see that $\bar{\Theta}_{t}:=e^{-\sigma^{2} t} \Theta_{t}$ is a unique solution of

$$
d \bar{\Theta}_{t}=2 \bar{\Theta}_{t}^{\lambda} d t, \quad \bar{\Theta}_{0}=x^{2}>0
$$

Hence

$$
d \Theta_{t}=e^{\sigma^{2} t}\left(2 \bar{\Theta}_{t}^{\lambda}+\sigma^{2} \bar{\Theta}_{t}\right) d t \geq\left(2 \Theta_{t}^{\lambda}+\sigma^{2} \Theta_{t}\right) d t
$$

Furthermore, by (1.2), Ito's formula and Jensen's inequality,

$$
\begin{aligned}
d \Phi_{t} & =d E\left[X_{t}^{2}\right] \\
& =E\left[2 X_{t}^{2 \lambda}-2 c_{t} X_{t}^{2}+\sigma^{2} X_{t}^{2}\right] d t \\
& \leq\left(2 \Phi_{t}^{\lambda}+\sigma^{2} \Phi_{t}\right) d t .
\end{aligned}
$$

Thus, we deduce $\Phi_{t} \leq \Theta_{t}$ and $\Phi_{0}=\Theta_{0}$, which implies (2.2).

Next, let $\left\{Y_{t}\right\}$ denote the solution $\left\{X_{t}^{y}\right\}$ of (1.2) with $Y_{0}=y$ and $y_{t}=Y_{t}^{1-\gamma}$. Then, by (2.4)

$$
d\left(x_{t}-y_{t}\right)=-(1-\gamma)\left(c_{t}+\frac{\sigma^{2}}{2} \gamma\right)\left(x_{t}-y_{t}\right) d t+(1-\gamma) \sigma\left(x_{t}-y_{t}\right) d B_{t}
$$

which implies

$$
x_{t}-y_{t}=\left(x_{0}-y_{0}\right) \exp \left\{-(1-\gamma)\left(\int_{0}^{t} c_{s} d s+\frac{\sigma^{2}}{2} \gamma t\right)+(1-\gamma) \sigma B_{t}-\frac{\sigma^{2}}{2}(1-\gamma)^{2} t\right\} \text {. }
$$

Setting $\beta=1 /(1-\gamma)>1$, we have

$$
\begin{aligned}
E\left[\left|x_{t}-y_{t}\right|^{\beta}\right] & \leq\left|x_{0}-y_{0}\right|^{\beta} E\left[\exp \left\{\sigma B_{t}-\frac{\sigma^{2}}{2} t\right\}\right] \\
& =\left|x^{1-\gamma}-y^{1-\gamma}\right|^{1 /(1-\gamma)} \leq|x-y| .
\end{aligned}
$$


By Young's inequality [11], for any $\varepsilon_{0}>0$,

$$
\begin{aligned}
\left|x^{\beta}-y^{\beta}\right| & \leq \beta\left(x^{\beta-1}+y^{\beta-1}\right)|x-y| \\
& \leq \beta\left[\frac{1}{\beta}\left(\frac{1}{\varepsilon_{0}}\right)^{\beta}|x-y|^{\beta}+\frac{\beta-1}{\beta}\left\{\varepsilon_{0}\left(x^{\beta-1}+y^{\beta-1}\right)\right\}^{\beta /(\beta-1)}\right] \\
& \leq\left(\frac{1}{\varepsilon_{0}}\right)^{\beta}|x-y|^{\beta}+(\beta-1)\left(2 \varepsilon_{0}\right)^{\beta /(\beta-1)}\left(x^{\beta}+y^{\beta}\right), \quad x, y \geq 0 .
\end{aligned}
$$

Hence, for any $\varepsilon>0$, we choose $C_{\varepsilon}>0$ such that

$$
\left|x^{\beta}-y^{\beta}\right| \leq C_{\varepsilon}|x-y|^{\beta}+\varepsilon\left(1+x^{\beta}+y^{\beta}\right), \quad x, y \geq 0 .
$$

Therefore, by (2.1) and (2.6), we have

$$
\begin{aligned}
E\left[\left|X_{t}-Y_{t}\right|\right] & =E\left[\left|x_{t}^{\beta}-y_{t}^{\beta}\right|\right] \\
& \leq C_{\varepsilon} E\left[\left|x_{t}-y_{t}\right|^{\beta}\right]+\varepsilon E\left[1+x_{t}^{\beta}+y_{t}^{\beta}\right] \\
& \leq C_{\varepsilon}|x-y|+\varepsilon E\left[1+X_{t}+Y_{t}\right] \\
& \leq C_{\varepsilon}|x-y|+\varepsilon\left\{1+2^{\beta}\left(t^{\beta}+x\right)+2^{\beta}\left(t^{\beta}+y\right)\right\}
\end{aligned}
$$

which implies (2.3).

Remark 2.1 The uniqueness for (1.2) is violated if $x=0$ and $c_{t}$ is deterministic since 0 and the limit process $\chi_{t}:=\lim _{x \rightarrow 0+} X_{t}^{x}$ satisfy (1.2) with $X_{0}=0$, and

$$
E\left[\chi_{t}^{1-\gamma}\right]=E\left[\int_{0}^{t}(1-\gamma)\left\{1-\left(c_{s}+\frac{\sigma^{2}}{2} \gamma\right) \chi_{s}^{1-\gamma}\right\} d s\right] \neq 0
$$

\section{Viscosity solutions of the HJB equation}

Definition 3.1 Let $u \in C(0, \infty)$. Then $u$ is called a viscosity solution of $(1.5)$ if the following relations are satisfied:

$$
\begin{aligned}
& \alpha u(x) \leq \frac{1}{2} \sigma^{2} x^{2} q+x^{\gamma} p+\tilde{U}(x, p), \quad \forall(p, q) \in J^{2,+} u(x), \forall x>0, \\
& \alpha u(x) \geq \frac{1}{2} \sigma^{2} x^{2} q+x^{\gamma} p+\tilde{U}(x, p), \quad \forall(p, q) \in J^{2,-} u(x), \forall x>0,
\end{aligned}
$$

where $J^{2,+} u(x)$ and $J^{2,-} u(x)$ are the second-order superjets and subjets [7].

Define the value function $u$ by

$$
u(x)=\sup _{c \in \mathcal{A}} E\left[\int_{0}^{\infty} e^{-\alpha t} U\left(c_{t} X_{t}\right) d t\right]
$$

where the supremum is taken over all systems $\left(\Omega, \mathcal{F}, P,\left\{\mathcal{F}_{t}\right\} ;\left\{B_{t}\right\},\left\{c_{t}\right\}\right)$.

In this section, we study the viscosity solution $u$ of the HJB equation (1.5). Due to Proposition 2.1, we can show the value function $u$ with the following properties. 
Lemma 3.1 We assume (1.4). Then we have

$$
0 \leq u(x) \leq \zeta(x):=x+\zeta_{0}, \quad x>0
$$

for some constant $\zeta_{0}>0$, and there exists $C_{\rho}>0$ for any $\rho>0$ such that

$$
|u(x)-u(y)| \leq C_{\rho}|x-y|+\rho(1+x+y), \quad x, y>0 .
$$

Proof Clearly, $u$ is nonnegative. By concavity, there is $\bar{C}>0$ such that

$$
U(x) \leq \alpha 2^{-1 /(1-\gamma)} x+\bar{C}, \quad x \geq 0
$$

By (1.1) and (2.1), we have

$$
\begin{aligned}
E\left[\int_{0}^{\infty} e^{-\alpha t} U\left(c_{t} X_{t}\right) d t\right] & \leq E\left[\int_{0}^{\infty} e^{-\alpha t}\left(\alpha 2^{-1 /(1-\gamma)} X_{t}+\bar{C}\right) d t\right] \\
& \leq \int_{0}^{\infty} e^{-\alpha t}\left\{\alpha\left(t^{1 /(1-\gamma)}+x\right)+\bar{C}\right\} d t \\
& =x+\alpha \int_{0}^{\infty} e^{-\alpha t} t^{1 /(1-\gamma)} d t+\bar{C} / \alpha
\end{aligned}
$$

which implies (3.2).

Now, let $\rho>0$ be arbitrary. By (1.4), there is $\delta>0$ such that $U(x) \leq \rho$ for all $x \in[0, \delta]$. Furthermore,

$$
|U(x)-U(y)| \leq U^{\prime}(\delta)|x-y|, \quad x, y \geq \delta
$$

Thus, we obtain a constant $C_{\rho}>0$, depending on $\rho>0$, such that

$$
|U(x)-U(y)| \leq C_{\rho}|x-y|+\rho, \quad \forall x, y \geq 0 .
$$

By (1.1), (2.3) and (3.4), we get

$$
\begin{aligned}
|u(x)-u(y)| & \leq \sup _{c \in \mathcal{A}} E\left[\int_{0}^{\infty} e^{-\alpha t}\left|U\left(c_{t} X_{t}\right)-U\left(c_{t} Y_{t}\right)\right| d t\right] \\
& \leq \sup _{c \in \mathcal{A}} E\left[\int_{0}^{\infty} e^{-\alpha t}\left\{C_{\rho}\left|X_{t}-Y_{t}\right|+\rho\right\} d t\right] \\
& \leq C_{\rho} \int_{0}^{\infty} e^{-\alpha t}\left\{C_{\varepsilon}|x-y|+\varepsilon\left(1+t^{1 /(1-\gamma)}+x+y\right)\right\} d t+\rho / \alpha \\
& \leq C\left\{C_{\rho} C_{\varepsilon}|x-y|+(\varepsilon+\rho)(1+x+y)\right\}, \quad x, y>0,
\end{aligned}
$$

where the constant $C>0$ is independent of $\varepsilon, \rho>0$. Replacing $\rho$ by $\rho / 2 C$ and choosing sufficiently small $\varepsilon>0$, we deduce (3.3).

Remark 3.1 The continuity of $u$ is immediate from (3.3).

Theorem 3.1 We assume (1.4). Then the value function $u$ is a viscosity solution of (1.5). 
Proof According to [12], the viscosity property of $u$ follows from the dynamic programming principle for $u$, that is,

$$
u(x)=\sup _{c \in \mathcal{A}} E\left[\int_{0}^{\tau} e^{-\alpha t} U\left(c_{t} X_{t}\right) d t+e^{-\alpha \tau} u\left(X_{\tau}\right)\right], \quad x>0
$$

for any bounded stopping time $\tau \geq 0$, where the supremum is taken over all systems $\left(\Omega, \mathcal{F}, P,\left\{\mathcal{F}_{t}\right\} ;\left\{B_{t}\right\},\left\{c_{t}\right\}\right)$. Let $\bar{u}(x)$ be the right-hand side of (3.6). Let $\tilde{X}_{t}=X_{t+r}$ and $\tilde{B}_{t}=$ $B_{t+r}-B_{r}$, when $\tau=r$ is non-random. Then we have

$$
d \tilde{X}_{t}=\left[\tilde{X}_{t}^{\gamma}-\tilde{c}_{t} \tilde{X}_{t}\right] d t+\sigma \tilde{X}_{t} d \tilde{B}_{t}, \quad \tilde{X}_{0}=X_{r}
$$

for the shifted process $\tilde{c}=\left\{\tilde{c}_{t}\right\}$ of $c$ by $r$, i.e., $\tilde{c}_{t}=c_{t+r}$. It is easy to see that

$$
e^{\alpha r} E\left[\int_{r}^{\infty} e^{-\alpha t} U\left(c_{t} X_{t}\right) d t \mid \mathcal{F}_{r}\right]=E\left[\int_{0}^{\infty} e^{-\alpha t} U\left(\tilde{c}_{t} \tilde{X}_{t}\right) d t \mid \mathcal{F}_{r}\right]=J_{X_{r}}(\tilde{c}) \quad \text { a.s. }
$$

with respect to the conditional probability $P\left(\cdot \mid \mathcal{F}_{r}\right)$. We take $\zeta_{1}>0$ such that $x^{\gamma} \leq \alpha x+\zeta_{1}$ and sufficiently large $\zeta_{0}>0$ to obtain

$$
-\alpha \zeta+\frac{1}{2} \sigma^{2} x^{2} \zeta^{\prime \prime}+x^{\gamma} \zeta^{\prime} \leq-\alpha \zeta_{0}+\zeta_{1} \leq 0
$$

By (3.2) in Lemma 3.1, Ito's formula and Doob's inequality, we have

$$
E\left[\sup _{0 \leq t \leq T} e^{-\alpha t} J_{X_{t}}(\tilde{c})\right] \leq E\left[\sup _{0 \leq t \leq T} e^{-\alpha t} \zeta\left(X_{t}\right)\right] \leq \zeta(x)+C, \quad T>0
$$

for some constant $C>0$. Hence, approximating $\tau$ by a sequence of countably valued stopping times, we see that

$$
E\left[e^{-\alpha \tau} J_{\tau}(\tilde{c})\right]=E\left[\int_{\tau}^{\infty} e^{-\alpha t} U\left(c_{t} X_{t}\right) d t\right] .
$$

Thus

$$
\begin{aligned}
J_{x}(c) & =E\left[\int_{0}^{\tau} e^{-\alpha t} U\left(c_{t} X_{t}\right) d t+\int_{\tau}^{\infty} e^{-\alpha t} U\left(c_{t} X_{t}\right) d t\right] \\
& \leq E\left[\int_{0}^{\tau} e^{-\alpha t} U\left(c_{t} X_{t}\right) d t+e^{-\alpha \tau} u\left(X_{\tau}\right)\right] .
\end{aligned}
$$

Taking the supremum, we deduce $u \leq \bar{u}$.

We shall show the reverse inequality in the case that $\tau=r$ is constant. For any $\varepsilon>0$, we consider a sequence $\left\{S_{j}: j=1, \ldots, n+1\right\}$ of disjoint subsets of $(0, \infty)$ such that

$$
\operatorname{diam}\left(S_{j}\right)<\delta, \quad \bigcup_{j=1}^{n} S_{j}=(0, R) \quad \text { and } \quad S_{n+1}=[R, \infty)
$$

for $\delta, R>0$ chosen later. We take $x_{j} \in S_{j}$ and $c^{(j)} \in \mathcal{A}$ such that

$$
u\left(x_{j}\right)-\varepsilon \leq J_{x_{j}}\left(c^{(j)}\right), \quad j=1, \ldots, n+1 .
$$


By the same argument as (3.5), we note that

$$
\left|J_{x}\left(c^{(j)}\right)-J_{y}\left(c^{(j)}\right)\right|+|u(x)-u(y)| \leq C_{\varepsilon}|x-y|+\frac{\varepsilon}{4}(1+x+y), \quad x, y>0
$$

for some constant $C_{\varepsilon}>0$. We choose $0<\delta<1$ such that $C_{\varepsilon} \delta<\varepsilon / 2$. Then we have

$$
\left|J_{x}\left(c^{(j)}\right)-J_{y}\left(c^{(j)}\right)\right|+|u(x)-u(y)| \leq \varepsilon(1+x), \quad x, y \in S_{j}, j=1,2, \ldots, n .
$$

Hence, by (3.7) and (3.8),

$$
\begin{aligned}
J_{X_{r}}\left(c^{(j)}\right) & =J_{X_{r}}\left(c^{(j)}\right)-J_{x_{j}}\left(c^{(j)}\right)+J_{x_{j}}\left(c^{(j)}\right) \\
& \geq-\varepsilon\left(1+X_{r}\right)+u\left(x_{j}\right)-\varepsilon \\
& \geq-2 \varepsilon\left(1+X_{r}\right)+u\left(X_{r}\right)-\varepsilon \\
& \geq-3 \varepsilon\left(1+X_{r}\right)+u\left(X_{r}\right) \quad \text { if } X_{r} \in S_{j}, j=1, \ldots, n
\end{aligned}
$$

By definition, we find $c \in \mathcal{A}$ such that

$$
\bar{u}(x)-\varepsilon \leq E\left[\int_{0}^{r} e^{-\alpha t} U\left(c_{t} X_{t}\right) d t+e^{-\alpha r} u\left(X_{r}\right)\right] .
$$

In view of $[10$, Chapter 6 , Theorem 1.1$]$, we can take $c, c^{(j)}$ on the same probability space. Define

$$
c_{t}^{r}=c_{t} 1_{\{t<r\}}+c_{t-r}^{(j)} 1_{\{r \leq t\}} \quad \text { if } X_{r} \in S_{j}, j=1, \ldots, n+1,
$$

where $1_{\{\cdot\}}$ denotes the indicator function. Then $\left\{c_{t}^{r}\right\}$ belongs to $\mathcal{A}$. Let $\left\{X_{t}^{r}\right\}$ be the solution of

$$
d X_{t}^{r}=\left[\left(X_{t}^{r}\right)^{\gamma}-c_{t}^{r} X_{t}^{r}\right] d t+\sigma X_{t}^{r} d B_{t}, \quad X_{0}^{r}=x>0
$$

Clearly, $X_{t}^{r}=X_{t}$ a.s. if $t<r$. Further, for each $j=1, \ldots, n+1$, we have on $\left\{X_{r} \in S_{j}\right\}$

$$
\begin{aligned}
X_{t+r}^{r} & =X_{r}+\int_{r}^{t+r}\left[\left(X_{s}^{r}\right)^{\gamma}-c_{s}^{r} X_{s}^{r}\right] d s+\int_{r}^{t+r} \sigma X_{s}^{r} d B_{s} \\
& =X_{r}+\int_{0}^{t}\left[\left(X_{s+r}^{r}\right)^{\gamma}-c_{s}^{(j)} X_{s+r}^{r}\right] d s+\int_{0}^{t} \sigma X_{s+r}^{r} d \tilde{B}_{s} \quad \text { a.s. }
\end{aligned}
$$

Hence, $X_{t+r}^{r}$ coincides with the solution $X_{t}^{(j)}$ of $(1.2)$ for $\left(\tilde{\Omega}, \tilde{\mathcal{F}}, \tilde{P},\left\{\tilde{\mathcal{F}}_{t}\right\} ;\left\{\tilde{B}_{t}\right\},\left\{c_{t}^{(j)}\right\}\right)$ a.s. on $\left\{X_{r} \in S_{j}\right\}$ with $X_{0}^{(j)}=X_{r}$. Thus, we get

$$
\begin{aligned}
J_{X_{r}}\left(\tilde{c}^{r}\right) & =E^{\tilde{P}}\left[\int_{0}^{\infty} e^{-\alpha t} U\left(c_{t+r}^{r} X_{t+r}^{r}\right) d t \mid \tilde{\mathcal{F}}_{r}\right] \\
& =E^{\tilde{P}}\left[\int_{0}^{\infty} e^{-\alpha t} U\left(c_{t}^{(j)} X_{t}^{(j)}\right) d t \mid \tilde{\mathcal{F}}_{r}\right] \\
& =J_{X_{r}}\left(c^{(j)}\right) \quad \text { a.s. on }\left\{X_{r} \in S_{j}\right\}, j=1, \ldots, n+1,
\end{aligned}
$$

where $E^{\tilde{P}}$ denotes the expectation with respect to $\tilde{P}$. 
Now, we fix $x>0$ and choose $R>0$, by (2.1), (2.2) and (3.1), such that

$$
\begin{aligned}
\sup _{c \in \mathcal{A}} E\left[u\left(X_{r}\right) 1_{\left\{X_{r} \geq R\right\}}\right] & \leq \sup _{c \in \mathcal{A}} E\left[\zeta\left(X_{r}\right) 1_{\left\{X_{r} \geq R\right\}}\right] \\
& \leq \sup _{c \in \mathcal{A}} \frac{1}{R} E\left[X_{r}^{2}+\zeta_{0} X_{r}\right] \\
& \leq \frac{C_{0}}{R}\left(1+x+x^{2}\right)<\varepsilon
\end{aligned}
$$

where the constant $C_{0}>0$ depends only on $r, \zeta_{0}$. By (3.9), (3.10) and (3.11), we have

$$
\begin{aligned}
E\left[\int_{r}^{\infty} e^{-\alpha t} U\left(c_{t}^{r} X_{t}^{r}\right) d t\right] & =E\left[E\left[\int_{r}^{\infty} e^{-\alpha t} U\left(c_{t}^{r} X_{t}^{r}\right) d t \mid \mathcal{F}_{r}\right]\right] \\
& =E\left[e^{-\alpha r} J_{X_{r}}\left(\tilde{c}^{r}\right)\right] \\
& =E\left[\sum_{j=1}^{n+1} e^{-\alpha r} J_{X_{r}}\left(c^{(j)}\right) 1_{\left\{X_{r} \in S_{j}\right\}}\right] \\
& \geq E\left[\sum_{j=1}^{n} e^{-\alpha r}\left\{u\left(X_{r}\right)-3 \varepsilon\left(1+X_{r}\right)\right\} 1_{\left\{X_{r} \in S_{j}\right\}}\right] \\
& \geq E\left[e^{-\alpha r}\left\{u\left(X_{r}\right)-u\left(X_{r}\right) 1_{\left\{X_{r} \geq R\right\}}\right\}\right]-3 \varepsilon E\left[1+X_{r}\right] \\
& \geq E\left[e^{-\alpha r} u\left(X_{r}\right)\right]-\varepsilon-3 \varepsilon C(1+x)
\end{aligned}
$$

for some constant $C>0$ independent of $\varepsilon$. Thus

$$
\begin{aligned}
u(x) & \geq E\left[\int_{0}^{r} e^{-\alpha t} U\left(c_{t}^{r} X_{t}^{r}\right) d t+\int_{r}^{\infty} e^{-\alpha t} U\left(c_{t}^{r} X_{t}^{r}\right) d t\right] \\
& \geq E\left[\int_{0}^{r} e^{-\alpha t} U\left(c_{t} X_{t}\right) d t+e^{-\alpha r} u\left(X_{r}\right)\right]-\varepsilon-3 \varepsilon C(1+x) \\
& \geq \bar{u}(x)-2 \varepsilon-3 \varepsilon C(1+x)
\end{aligned}
$$

Letting $\varepsilon \rightarrow 0$, we get $\bar{u} \leq u$.

In the general case, by the above argument, we note that

$$
\begin{aligned}
u\left(X_{r}\right) & =u\left(\tilde{X}_{0}\right) \geq E\left[\int_{0}^{s} e^{-\alpha t} U\left(\tilde{c}_{t} \tilde{X}_{t}\right) d t+e^{-\alpha s} u\left(\tilde{X}_{s}\right) \mid \mathcal{F}_{r}\right] \\
& =E\left[\int_{0}^{s} e^{-\alpha t} U\left(c_{t+r} X_{t+r}\right) d t+e^{-\alpha s} u\left(X_{s+r}\right) \mid \mathcal{F}_{r}\right] \quad \text { a.s. } s, r \geq 0 .
\end{aligned}
$$

Hence $\left\{e^{-\alpha s} u\left(X_{s}\right)+\int_{0}^{s} e^{-\alpha t} U\left(c_{t} X_{t}\right) d t\right\}$ is a supermartingale. By the optional sampling theorem,

$$
u\left(X_{0}\right) \geq E\left[\int_{0}^{\tau} e^{-\alpha t} U\left(c_{t} X_{t}\right) d t+e^{-\alpha \tau} u\left(X_{\tau}\right) \mid \mathcal{F}_{0}\right] \quad \text { a.s. }
$$

Taking the expectation and then the supremum over $\mathcal{A}$, we conclude that $\bar{u} \leq u$. Noting the continuity of $u$, we obtain (3.6). 


\section{Classical solutions}

In this section, using the viscosity solutions technique, we show the $C^{2}$-regularity of the viscosity solution $u$ of (1.5). For any fixed $0<a<b$, we consider the boundary value problem

$$
\alpha w=\frac{1}{2} \sigma^{2} x^{2} w^{\prime \prime}+x^{\gamma} w^{\prime}+\tilde{U}\left(x, w^{\prime}\right) \quad \text { in }(a, b),
$$

with boundary condition

$$
w(a)=u(a), \quad w(b)=u(b),
$$

given by $u$.

Proposition 4.1 Let $w_{i} \in C[a, b], i=1,2$, be two viscosity solutions of (3.1), (4.2). Then, under (1.4), we have

$$
w_{1}=w_{2} .
$$

Proof It is sufficient to show that $w_{1} \leq w_{2}$. Suppose that there exists $x_{0} \in[a, b]$ such that $w_{1}\left(x_{0}\right)-w_{2}\left(x_{0}\right)>0$. Clearly, by (4.2), $x_{0} \neq a, b$, and we find $\bar{x} \in(a, b)$ such that

$$
\varrho:=\sup _{x \in[a, b]}\left\{w_{1}(x)-w_{2}(x)\right\}=w_{1}(\bar{x})-w_{2}(\bar{x})>0 .
$$

Define

$$
\Psi_{k}(x, y)=w_{1}(x)-w_{2}(y)-\frac{k}{2}|x-y|^{2}
$$

for $k>0$. Then there exists $\left(x_{k}, y_{k}\right) \in[a, b]^{2}$ such that

$$
\Psi_{k}\left(x_{k}, y_{k}\right)=\sup _{(x, y) \in[a, b]^{2}} \Psi_{k}(x, y) \geq \Psi_{k}(\bar{x}, \bar{x})=\varrho,
$$

from which

$$
\frac{k}{2}\left|x_{k}-y_{k}\right|^{2}<w_{1}\left(x_{k}\right)-w_{2}\left(y_{k}\right) .
$$

Thus

$$
\left|x_{k}-y_{k}\right| \rightarrow 0 \text { as } k \rightarrow \infty \text {. }
$$

Furthermore, by the definition of $\left(x_{k}, y_{k}\right)$,

$$
\Psi_{k}\left(x_{k}, y_{k}\right) \geq \Psi_{k}\left(x_{k}, x_{k}\right) .
$$

Hence, by uniform continuity

$$
\begin{aligned}
\frac{k}{2}\left|x_{k}-y_{k}\right|^{2} & \leq w_{2}\left(x_{k}\right)-w_{2}\left(y_{k}\right) \leq \sup _{|x-y| \leq \rho}\left|w_{2}(x)-w_{2}(y)\right| \\
& \rightarrow 0 \quad \text { as } k \rightarrow \infty \text { and then } \rho \rightarrow 0 .
\end{aligned}
$$


By (4.3), (4.4) and (4.5), extracting a subsequence, we have

$$
\left(x_{k}, y_{k}\right) \rightarrow(\tilde{x}, \tilde{x}) \in(a, b)^{2} \quad \text { as } k \rightarrow \infty .
$$

Now, we may consider that $\left(x_{k}, y_{k}\right) \in(a, b)^{2}$ for sufficiently large $k$. Applying Ishii's lemma [7] to $\Psi_{k}(x, y)$, we obtain $X, Y \in \mathbf{R}$ such that

$$
\begin{aligned}
& \left(k\left(x_{k}-y_{k}\right), X\right) \in \bar{J}^{2,+} w_{1}\left(x_{k}\right), \\
& \left(k\left(x_{k}-y_{k}\right), Y\right) \in \bar{J}^{2,-} w_{2}\left(y_{k}\right), \\
& \left(\begin{array}{cc}
X & 0 \\
0 & -Y
\end{array}\right) \leq 3 k\left(\begin{array}{cc}
1 & -1 \\
-1 & 1
\end{array}\right) .
\end{aligned}
$$

By Definition 3.1,

$$
\begin{aligned}
& \alpha w_{1}\left(x_{k}\right) \leq \frac{1}{2} \sigma^{2} x_{k}^{2} X+x_{k}^{\gamma} \mu+\tilde{U}\left(x_{k}, \mu\right), \\
& \alpha w_{2}\left(y_{k}\right) \geq \frac{1}{2} \sigma^{2} y_{k}^{2} Y+y_{k}^{\gamma} \mu+\tilde{U}\left(y_{k}, \mu\right),
\end{aligned}
$$

where $\mu=k\left(x_{k}-y_{k}\right)$. Putting these inequalities together, we get

$$
\begin{aligned}
\alpha\left\{w_{1}\left(x_{k}\right)-w_{2}\left(y_{k}\right)\right\} & \leq \frac{1}{2} \sigma^{2}\left(x_{k}^{2} X-y_{k}^{2} Y\right)+\left(x_{k}^{\gamma}-y_{k}^{\gamma}\right) \mu+\left\{\tilde{U}\left(x_{k}, \mu\right)-\tilde{U}\left(y_{k}, \mu\right)\right\} \\
& \equiv I_{1}+I_{2}+I_{3}, \quad \text { say. }
\end{aligned}
$$

By (4.5) and (4.7), it is clear that

$$
I_{1}=\frac{\sigma^{2}}{2}\left(x_{k}^{2} X-y_{k}^{2} Y\right) \leq \frac{\sigma^{2}}{2} 3 k\left(x_{k}-y_{k}\right)^{2} \rightarrow 0 \quad \text { as } k \rightarrow \infty .
$$

Also, by (4.5)

$$
I_{2}=k\left(x_{k}^{\gamma}-y_{k}^{\gamma}\right)\left(x_{k}-y_{k}\right) \leq k \gamma a^{\gamma-1}\left|x_{k}-y_{k}\right|^{2} \rightarrow 0 \quad \text { as } k \rightarrow \infty .
$$

By (1.6), (3.4), (4.5) and (4.6), we have

$$
\begin{aligned}
I_{3} & \leq \max _{0 \leq c \leq 1}\left|U\left(c x_{k}\right)-U\left(c y_{k}\right)\right|+\left|x_{k}-y_{k}\right||\mu| \\
& \leq C_{\rho}\left|x_{k}-y_{k}\right|+\rho+k\left|x_{k}-y_{k}\right|^{2} \\
& \rightarrow 0 \text { as } k \rightarrow \infty \text { and then } \rho \rightarrow 0 .
\end{aligned}
$$

Consequently, by (4.6), we deduce that

$$
\alpha \varrho \leq \alpha\left\{w_{1}(\tilde{x})-w_{2}(\tilde{x})\right\} \leq 0,
$$

which is a contradiction.

Theorem 4.1 We assume (1.4). Then there exists a solution $u \in C^{2}(0, \infty)$ of $(1.5)$. 
Proof For any $0<a<b$, we recall the boundary value problem (4.1), (4.2). Since

$$
U(0) \leq U^{\prime}(x)(0-x)+U(x), \quad x>0,
$$

we have

$$
K_{0}:=\sup _{0<x \leq a} x U^{\prime}(x)<\infty
$$

Hence, by (1.4)

$$
\begin{aligned}
\left|U\left(c x_{1}\right)-U\left(c x_{2}\right)\right| & \leq c U^{\prime}(c a)\left|x_{1}-x_{2}\right| \\
& \leq \frac{K_{0}}{a}\left|x_{1}-x_{2}\right|, \quad x_{1}, x_{2} \in[a, b], 0 \leq c \leq 1 .
\end{aligned}
$$

Also, by (1.6)

$$
\begin{aligned}
\left|\tilde{U}\left(x_{1}, y_{1}\right)-\tilde{U}\left(x_{2}, y_{2}\right)\right| & \leq \max _{0 \leq c \leq 1}\left|U\left(c x_{1}\right)-U\left(c x_{2}\right)\right|+\left|x_{1} y_{1}-x_{2} y_{2}\right| \\
& \leq \frac{K_{0}}{a}\left|x_{1}-x_{2}\right|+\left|x_{1}-x_{2}\right|\left|y_{1}\right|+b\left|y_{1}-y_{2}\right|, \quad y_{1}, y_{2}>0 .
\end{aligned}
$$

Thus the nonlinear term of (4.1) is Lipschitz. By uniform ellipticity, a standard theory of nonlinear elliptic equations yields that there exists a unique solution $w \in C^{2}(a, b) \cap C[a, b]$ of (4.1), (4.2). For details, we refer to [13, Theorem 17.18] and [14, Chapter 5, Theorem 3.7]. Clearly, by Theorem 3.1, $u$ is a viscosity solution of (4.1), (4.2). Therefore, by Proposition 4.1, we have $w=u$ and $u$ is smooth. Since $a, b$ are arbitrary, we obtain the assertion.

\section{Optimal consumption}

In this section, we give a synthesis of the optimal policy $c^{*}=\left\{c_{t}^{*}\right\}$ for the optimization problem (1.4) subject to (1.2). We consider the stochastic differential equation

$$
d X_{t}^{*}=\left[\left(X_{t}^{*}\right)^{\gamma}-\eta\left(X_{t}^{*}\right) X_{t}^{*}\right] d t+\sigma X_{t}^{*} d B_{t}, \quad X_{0}^{*}=x>0,
$$

where $\eta(x)=I\left(x, u^{\prime}(x)\right)$ and $I(x, y)$ denotes the maximizer of (1.6) for $x, y>0$, i.e.,

$$
I(x, y)= \begin{cases}\left(U^{\prime}\right)^{-1}(y) / x & \text { if } U^{\prime}(x) \leq y \\ 1 & \text { otherwise }\end{cases}
$$

Our objective is to prove the following.

Theorem 5.1 We assume (1.4). Then the optimal consumption policy $\left\{c_{t}^{*}\right\}$ is given by

$$
c_{t}^{*}=\eta\left(X_{t}^{*}\right) .
$$

To obtain the optimal consumption policy $\left\{c_{t}^{*}\right\}$, we should study the properties of the value function $u$ and the existence of strong solution $\left\{X_{t}^{*}\right\}$ of (5.1). We need the following lemmas. 
Lemma 5.1 Under (1.4), the value function $u$ is concave. In addition, we have

$$
\begin{aligned}
& u^{\prime}(x)>0 \quad \text { for } x>0, \\
& u^{\prime}(0+)=\infty .
\end{aligned}
$$

Proof Let $x_{i}>0, i=1,2$. For any $\varepsilon>0$, there exists $c^{(i)} \in \mathcal{A}$ such that

$$
u\left(x_{i}\right)-\varepsilon<E\left[\int_{0}^{\infty} e^{-\alpha t} U\left(c_{t}^{(i)} X_{t}^{(i)}\right) d t\right]
$$

where $\left\{X_{t}^{(i)}\right\}$ is the solution of (1.2) corresponding to $c^{(i)}$ with $X_{0}^{(i)}=x_{i}$. Let $0 \leq \xi \leq 1$, and we set

$$
\bar{c}_{t}=\frac{\xi c_{t}^{(1)} X_{t}^{(1)}+(1-\xi) c_{t}^{(2)} X_{t}^{(2)}}{\xi X_{t}^{(1)}+(1-\xi) X_{t}^{(2)}},
$$

which belongs to $\mathcal{A}$. Define $\left\{\bar{X}_{t}\right\}$ and $\left\{\tilde{X}_{t}\right\}$ by

$$
\begin{aligned}
& d \bar{X}_{t}=\left[\left(\bar{X}_{t}\right)^{\gamma}-\bar{c}_{t} \bar{X}_{t}\right] d t+\sigma \bar{X}_{t} d B_{t}, \quad \bar{X}_{0}=\xi x_{1}+(1-\xi) x_{2}, \\
& \tilde{X}_{t}=\xi X_{t}^{(1)}+(1-\xi) X_{t}^{(2)} .
\end{aligned}
$$

By concavity,

$$
\tilde{X}_{t} \leq \xi x_{1}+(1-\xi) x_{2}+\int_{0}^{t}\left[\left(\tilde{X}_{s}\right)^{\gamma}-\bar{c}_{s} \tilde{X}_{s}\right] d s+\int_{0}^{t} \sigma \tilde{X}_{s} d B_{s} \quad \text { a.s. }
$$

By the comparison theorem, we have

$$
\tilde{X}_{t} \leq \bar{X}_{t} \quad \text { for all } t \geq 0 \text { a.s. }
$$

Thus, by (1.4)

$$
\begin{aligned}
u\left(\xi x_{1}+(1-\xi) x_{2}\right) & \geq E\left[\int_{0}^{\infty} e^{-\alpha t} U\left(\bar{c}_{t} \bar{X}_{t}\right) d t\right] \geq E\left[\int_{0}^{\infty} e^{-\alpha t} U\left(\bar{c}_{t} \tilde{X}_{t}\right) d t\right] \\
& =E\left[\int_{0}^{\infty} e^{-\alpha t} U\left(\xi c_{t}^{(1)} X_{t}^{(1)}+(1-\xi) c_{t}^{(2)} X_{t}^{(2)}\right) d t\right] \\
& \geq \xi E\left[\int_{0}^{\infty} e^{-\alpha t} U\left(c_{t}^{(1)} X_{t}^{(1)}\right) d t\right]+(1-\xi) E\left[\int_{0}^{\infty} e^{-\alpha t} U\left(c_{t}^{(2)} X_{t}^{(2)}\right) d t\right] \\
& \geq \xi u\left(x_{1}\right)+(1-\xi) u\left(x_{2}\right)-\varepsilon
\end{aligned}
$$

Therefore, letting $\varepsilon \rightarrow 0$, we obtain the concavity of $u$.

To prove (5.4), by Theorem 4.1, we recall that $u$ is smooth. Furthermore, we get $u^{\prime}(x) \geq 0$ for $x>0$. If not, then $u^{\prime}\left(a_{0}\right)<0$ for some $a_{0}>0$. By concavity,

$$
0 \leq u(x) \leq u^{\prime}\left(a_{0}\right)\left(x-a_{0}\right)+u\left(a_{0}\right) \rightarrow-\infty \quad \text { as } x \rightarrow \infty,
$$


which is a contradiction. Suppose that $u^{\prime}(z)=0$ for some $z>0$. Then, by concavity, we have $u^{\prime}(x)=0$ for all $x \geq z$. Hence, by (1.5) and (1.6),

$$
\alpha u(z)=\alpha u(x)=\tilde{U}(x, 0)=U(x), \quad x \geq z .
$$

This is contrary to (1.4). Thus, we obtain (5.4).

Next, by definition, we have

$$
0<E\left[\int_{0}^{\infty} e^{-\alpha t} U\left(\check{X}_{t}\right) d t\right] \leq u(x), \quad x>0
$$

where $\left\{\check{X}_{t}\right\}$ is the solution of (1.2) corresponding to $c_{t}=1$. As in (2.7), the limit process $\check{X}_{t}:=\lim _{x \rightarrow 0+} \check{X}_{t}$ is different from 0 . Hence

$$
0<E\left[\int_{0}^{\infty} e^{-\alpha t} U\left(\check{\chi}_{t}\right) d t\right] \leq u(0+)
$$

Suppose that $u^{\prime}(0+)<\infty$. By $(1.5)$ and concavity, we get $u(0+)=0$, which is a contradiction. This implies (5.5).

Lemma 5.2 Under (1.4), there exists a unique positive strong solution $\left\{X_{t}^{*}\right\}$ of (5.1).

Proof Let $\left\{N_{t}\right\}$ be the solution of (1.2) corresponding to $c_{t}=0$. We can take the Brownian motion $\left\{B_{t}\right\}$ on the canonical probability space [4, p.71]. Since $0 \leq \eta \leq 1$, the probability measure $\hat{P}$ is defined by

$$
d \hat{P} / d P=\exp \left\{-\int_{0}^{t} \eta\left(N_{s}\right) / \sigma d B_{s}-\frac{1}{2} \int_{0}^{t}\left(\eta\left(N_{s}\right) / \sigma\right)^{2} d s\right\}
$$

for every $t \geq 0$. Girsanov's theorem yields that

$$
\hat{B}_{t}:=B_{t}+\int_{0}^{t} \eta\left(N_{s}\right) / \sigma d s \quad \text { is a Brownian motion under } \hat{P} .
$$

Hence

$$
d N_{t}=\left[\left(N_{t}\right)^{\gamma}-\eta\left(N_{t}\right) N_{t}\right] d t+\sigma N_{t} d \hat{B}_{t} \quad \text { under } \hat{P} .
$$

Thus, (5.1) admits a weak solution.

Now, by (5.2), we have

$$
\eta(x) x=\min \left\{\left(U^{\prime}\right)^{-1} \circ u^{\prime}(x), x\right\} .
$$

Hence, by (1.4) and concavity,

$$
\frac{d}{d x}\left(U^{\prime}\right)^{-1} \circ u^{\prime}(x)=\frac{u^{\prime \prime}(x)}{U^{\prime \prime} \circ\left(U^{\prime}\right)^{-1} \circ u^{\prime}(x)} \geq 0 .
$$

Thus, $\eta(x) x$ is nondecreasing on $(0, \infty)$. We rewrite (5.1) as the form of (2.4) to obtain $X_{t}^{*}>0$ a.s. Then we see that the pathwise uniqueness holds for (5.1). Therefore, by the 
Yamada-Watanabe theorem [10], we deduce that (5.1) admits a unique strong solution $\left\{X_{t}^{*}\right\}$.

Proof of Theorem 5.1 Since $\left\{c_{t}^{*}\right\}$ satisfies (1.1), it belongs to $\mathcal{A}$. By Lemma 5.2, we note that

$$
0<u^{\prime}(x) x \leq u(x)-u(0+)<u(x), \quad x>0 .
$$

Hence, by (2.2) and (3.2),

$$
\begin{aligned}
E\left[\int_{0}^{t}\left\{e^{-\alpha s} u^{\prime}\left(X_{s}^{*}\right) X_{s}^{*}\right\}^{2} d s\right] & \leq E\left[\int_{0}^{t}\left\{e^{-\alpha s} u\left(X_{s}^{*}\right)\right\}^{2} d s\right] \\
& \leq E\left[\int_{0}^{t} e^{-\alpha s} \zeta\left(X_{s}^{*}\right)^{2} d s\right]<\infty .
\end{aligned}
$$

This yields that $\left\{\int_{0}^{t} e^{-\alpha s} u^{\prime}\left(X_{s}^{*}\right) X_{s}^{*} d B_{s}\right\}$ is a martingale. By (1.6), (5.3) and Ito's formula,

$$
\begin{aligned}
E\left[e^{-\alpha t} u\left(X_{t}^{*}\right)\right]= & u(x)+E\left[\int _ { 0 } ^ { t } e ^ { - \alpha s } \left\{-\alpha u\left(X_{s}^{*}\right)+\left(X_{s}^{*}\right)^{\gamma} u^{\prime}\left(X_{s}^{*}\right)\right.\right. \\
& \left.\left.-c_{s}^{*} X_{s}^{*} u^{\prime}\left(X_{s}^{*}\right)+\frac{1}{2} \sigma^{2}\left(X_{s}^{*}\right)^{2} u^{\prime \prime}\left(X_{s}^{*}\right)\right\} d s\right] \\
= & u(x)-E\left[\int_{0}^{t} e^{-\alpha s} U\left(c_{s}^{*} X_{s}^{*}\right) d s\right] .
\end{aligned}
$$

By (2.1) and (3.2), it is clear that

$$
\begin{aligned}
E\left[e^{-\alpha t} u\left(X_{t}^{*}\right)\right] & \leq E\left[e^{-\alpha t} \zeta\left(X_{t}^{*}\right)\right] \\
& \leq e^{-\alpha t}\left\{(1-\gamma) t+x^{(1-\gamma)}\right\}^{1 /(1-\gamma)}+e^{-\alpha t} \zeta_{0} \rightarrow 0 \quad \text { as } t \rightarrow \infty .
\end{aligned}
$$

Letting $t \rightarrow \infty$, we deduce

$$
E\left[\int_{0}^{\infty} e^{-\alpha t} U\left(c_{t}^{*} X_{t}^{*}\right) d t\right]=u(x)
$$

By the same calculation as above, we obtain

$$
E\left[\int_{0}^{\infty} e^{-\alpha t} U\left(c_{t} X_{t}\right) d t\right] \leq u(x)
$$

for any $c \in \mathcal{A}$. The proof is complete.

Remark 5.1 From the proof of Theorem 5.1, it follows that the solution $u$ of the HJB equation (1.5) coincides with the value function. This implies that the uniqueness holds for (1.5). 


\section{Acknowledgements}

I would like to thank Professor H Morimoto for his useful help. The research was supported by the National Natural Science Foundation of China (11171275) and the Fundamental Research Funds for the Central Universities (XDJK2012C045).

Received: 10 June 2014 Accepted: 24 September 2014 Published: 13 Oct 2014

\section{References}

1. Merton, RC: An asymptotic theory of growth under uncertainty. Rev. Econ. Stud. 42, 375-393 (1975)

2. Baten, MA, Kamil, AA: Optimal consumption in a stochastic Ramsey model with Cobb-Douglas production function. Int. J. Math. Math. Sci. (2013). doi:10.1155/2013/684757

3. Barro, RJ, Sala-i-Martin, X: Economic Growth, 2nd edn. MIT Press, Cambridge (2004)

4. Kamien, MI, Schwartz, NL: Dynamic Optimization, 2nd edn. North-Holland, Amsterdam (1991)

5. Sethi, SP, Thompson, GL: Optimal Control Theory, 2nd edn. Kluwer Academic, Boston (2000)

6. Morimoto, $\mathrm{H}$, Zhou, $\mathrm{XY}$ : Optimal consumption in a growth model with the Cobb-Douglas production function. SIAM J. Control Optim. 47(6), 2991-3006 (2009)

7. Crandall, MG, Ishii, H, Lions, PL: User's guide to viscosity solutions of second order partial differential equations. Bull. Am. Math. Soc. 27, 1-67 (1992)

8. Koike, I: A Beginner's Guide to Theory of Viscosity Solutions. MSJ Memoirs. Math. Soc. Japan, Tokyo (2004)

9. Darling, RWR, Pardoux, E: Backwards SDE with random terminal time and applications to semilinear elliptic PDE. Ann. Probab. 25, 1135-1159 (1997)

10. Ikeda, N, Watanabe, S: Stochastic Differential Equations and Diffusion Processes. North-Holland, Amsterdam (1981)

11. Pales, Z: A general version of Young's inequality. Arch. Math. 58(4), 360-365 (1992)

12. Fleming, WH, Soner, HM: Controlled Markov Processes and Viscosity Solutions. Springer, New York (1993)

13. Gilbarg, D, Trudinger, NS: Elliptic Partial Differential Equations of Second Order. Springer, Berlin (1983)

14. Morimoto, H: Stochastic Control and Mathematical Modeling: Applications in Economics. Cambridge University Press, Cambridge (2010)

\section{Submit your manuscript to a SpringerOpen ${ }^{\circ}$ journal and benefit from:}

- Convenient online submission

Rigorous peer review

- Immediate publication on acceptance

- Open access: articles freely available online

- High visibility within the field

- Retaining the copyright to your article 